\title{
Alpha, beta: The rhythm of the attentional blink
}

\author{
Kimron L. Shapiro ${ }^{1} \cdot$ Simon Hanslmayr ${ }^{1}$ - James T. Enns ${ }^{2}$ • Alejandro Lleras ${ }^{3}$
}

Published online: 7 March 2017

(C) The Author(s) 2017. This article is published with open access at Springerlink.com

\begin{abstract}
Extant theories of the attentional blink propose that the most critical factor in determining second target accuracy is the time that elapses between the first and second targets. We report that this conclusion has overlooked an equally important determinant, namely, the frequency of the entraining stream in which these targets are embedded. Specifically, we show in two experiments that the signature of the attentional blink - second target accuracy that increases with intertarget lag - is significantly larger for entraining streams that are in the alpha-beta frequency range, relative to streams that are slower (theta) or faster (gamma). This finding ties the attentional blink critically, for the first time, to these two prominent oscillation frequencies that are known to be involved in the control of human attention and consciousness.
\end{abstract}

Keywords Attentional blink · Attention · Oscillations · Alpha $\cdot$ Beta

One of the ubiquitous findings in cognitive psychology is that humans have severe difficulty processing two visual inputs in rapid succession. This processing limitation is strikingly evident in the attentional blink task (AB; Raymond et al., 1992), which reveals that only the first of the two targets reaches awareness unless they are separated by at least half a second.

Kimron L. Shapiro

k.1.shapiro@bham.ac.uk

1 School of Psychology, University of Birmingham, Edgbaston Birmingham B15 2TT, UK

2 Department of Psychology, University of British Columbia, 2136 West Mall, Vancouver, BC V6T 1Z4, Canada

3 Psychology Department, University of Illinois, 603 East Daniel St., Urbana-Champaign, IL 61820, USA
Since its discovery, the AB has been replicated hundreds of times and is often-cited textbook knowledge. Remarkably, virtually all of the hundreds of $\mathrm{AB}$ studies to date have embedded targets in a visual stream presented at approximately $10-16 \mathrm{~Hz}$, placing it squarely in the oscillatory range of the alpha-beta complex (Scheeringa et al., 2011). Here we show that the attentional blink is largest when the stimuli are presented in two neighbouring frequency bands; alpha, the most dominant oscillatory frequency in the human brain (Berger, 1929), and beta, the nearby band whose effects often mirror it. Although historically these frequencies have been treated separately, recent EEG/MEG studies reveal that alpha $(\sim 10 \mathrm{~Hz})$ and beta $(\sim 15 \mathrm{~Hz})$ oscillations typically both decrease in amplitude during perception tasks, and that their dynamics are tightly correlated (Scheeringa et al., 2011).

The current finding is important first because the attentional blink has been linked to failures of conscious access, not failures of perceptual processing at an implicit level (Luck et al., 1996). The demonstration in the present study that the $\mathrm{AB}$ is dependent on specific stream rates thus ties conscious awareness very directly to these prominent rhythms in the brain. Second, extant theories of the AB all propose that the critical factor in predicting a blink is elapsed time (lag) between the presentation of the first and second targets. The main idea is that the lag-dependent second target deficit is either an index of the time that is required for the first target to be consolidated into working memory (Chun \& Potter, 1995), or the time that is required to regain control over the task set that has inadvertently been waylaid by the presence of distractor items in the stream (Di Lollo, Kawahara, Ghorashi, \& Enns, 2005; Olivers \& Meeter, 2008).

Here we test an alternative hypothesis that the secondtarget deficit in the attentional blink task is dependent on brain oscillations generated by the rate of the stream in which the two targets are embedded. To do this, we hold lag constant at a 
duration that is known to produce the $\mathrm{AB}$ in hundreds of past studies. We then show that despite this constancy, the AB rises and falls as a function of a factor that has heretofore not been systematically manipulated. This is the frequency of visual stimulation, caused by the rate of the stream in which these targets are embedded and which has been shown to cause entrainment in the visual cortex matching the rate of stimulus presentation (Herrmann, 2001).

It has long been known that brain oscillations play a crucial role in controlling the sleep-wakefulness cycle generally, and specifically, that they are relevant in guiding the timing of information processing (Buzsáki \& Draguhn, 2004). Oscillations in the alpha range are especially important for perception (Hanslmayr, Gross, Klimesch, \& Shapiro, 2011; Mathewson et al., 2012), with high amplitude alpha oscillation just prior to the onset of a stimulus associated with impaired target detection (Hanslmayr, Staudigl, \& Klimesch, 2007). Moreover, when the observer is engaged in target detection processing, the brain appears to be driven to levels of higher alpha synchronization (Mima et al., 2001). With regard to the AB then, there appear to be two factors that may link second target accuracy to neural oscillations in the alpha-beta range: (1) the rate of the leading stream of items prior to the presentation of targets and (2) the observer's level of engagement with first target processing. The direct implication is that the ubiquitous tendency in past research to present stream items at or near $10 \mathrm{~Hz}$ may have masked the observation that the $\mathrm{AB}$ is enhanced by stimulus streams that are presented in the alphabeta range (MacLean, Arnell, \& Cote, 2012).

In this study we measured the attentional blink at three different target lags (jittered lags of $\sim 100 \mathrm{~ms}, \sim 300 \mathrm{~ms}$, and $\sim 700 \mathrm{~ms}$ ) and at four different entrainment frequencies (Theta: 6.26 Hz, Alpha: 10.3 Hz, Beta: $16.0 \mathrm{~Hz}$, and Gamma: $36.0 \mathrm{~Hz}$ ). These specific frequencies were carefully selected so that the overlapping harmonics with one another were minimized (Pletzer, Kerschbaum, \& Klimesch, 2010). At the end of every trial, observers reported the identity of two letters that were presented in streams of black letters. According to extant theories of the $\mathrm{AB}$, intertarget lag should be the primary determinant of second target accuracy. The alternative hypothesis we pursued was that this lag-dependent finding would be larger for entrainment frequencies in the alpha-beta range.

\section{Experiment 1}

This experiment tested observers in a task previously shown to produce an AB (e.g. Arnell, Howe, Joanisse, \& Klein, 2006), where two red letters are embedded in streams of black letters are to be reported on every trial. The comparison of different presentation rates enabled us to test our main hypothesis that the $\mathrm{AB}$ will be most prominent at alpha and beta frequencies.

\section{Method}

Participants Twenty-four naïve participants in the dual-task experiment had a mean age of 19.5 years (21 females and three males), with normal or corrected-to-normal colour vision. This sample size was determined a priori as the minimum required to fully counterbalance the order of frequency blocks with all 24 possible order permutations across participants, and was larger than necessary to measure the $\mathrm{AB}$ according to the extant literature. Participants were to be excluded if accuracy over all conditions was below $50 \%$ (chance $=$ $33 \%$ ), and by this criterion no participants were excluded. The experiment was conducted at the University of Birmingham, with ethical approval obtained in accordance with APA and university policies.

Stimuli and procedure The relationship between lag and entrainment frequency in the twelve conditions tested in this experiment are shown in Fig. 1.

Displays were viewed at a distance of $70 \mathrm{~cm}$ and presented on a $33 \times 60 \mathrm{~cm}$ ASUS VG278HE LCD Monitor, with a $144 \mathrm{~Hz}$ refresh rate. Stream letters measured approximately $1.2 \mathrm{~cm}$ in height $\left(1.0^{\circ}\right.$ visual angle $)$ and $1.2 \mathrm{~cm}$ in width $\left(1.0^{\circ}\right.$ visual angle) and were presented for $21 \mathrm{~ms}$ in all conditions. Black distractor letters were presented for $\sim 1$ second period before the presentation of $\mathrm{T} 1$, and for a $\sim 550 \mathrm{~ms}$ period after $\mathrm{T} 2$, with slight variations to maintain in-phase relationships throughout the entire stimulus stream. The T1 to T2 intervals, labelled as Lag 100, Lag 300, and Lag 700 in the tables and figures, were also jittered between frequency conditions in order to maintain phase. The exact values are reported in Table 1. The number of items between $\mathrm{T} 1$ and $\mathrm{T} 2$ are reported in Table 2. The cue to report the target letter was a black asterisk and it was presented immediately following each letter stream. At the end of every trial, observers reported which of six possible alternative red letters (T1: B, G, S; T2: X, K, Y) were presented. Participants were instructed to select one of the three possible T1 items first, and subsequently to select one of the three possible T2 items. The program would not continue until a valid selection was made for each in turn. Thus, chance performance was $33 \%$ with no possibility of order reversals. The four presentation frequency conditions (6.26 Hz, $10.3 \mathrm{~Hz}, 16.0 \mathrm{~Hz}, 36 \mathrm{~Hz}$ ) were presented to all participants, separated into individual blocks of 81 trials each (324 per participant), with the order of the frequency blocks counterbalanced. As practice trials were not given, the first five trials of each block were excluded from further analysis.

\section{Results}

Table 3 shows the mean correct responses and standard errors for first target accuracy (T1) and for second target accuracy conditional on a correct response to T1 (T2|T1). Figure 2 


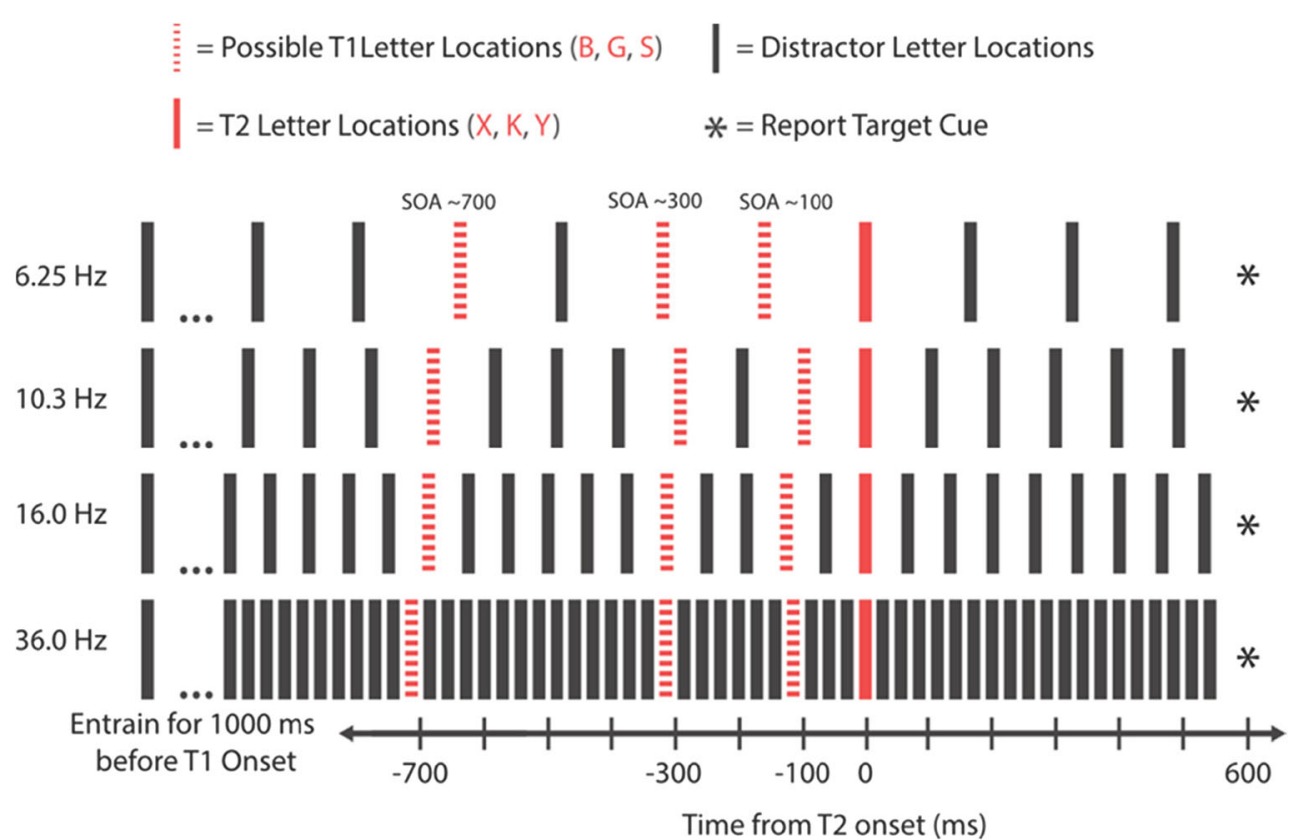

Fig. 1 Temporal sequence of events in Experiment 1. Two targets were embedded in distractor streams of different underlying frequencies: theta, alpha, beta, and gamma. Participant's task was to report the two targets embedded in the stream. Temporal lag between targets was varied from

shows the results for our experimental hypothesis, which revealed a striking outcome. The magnitude of the attentional blink, measured as the difference in T2|T1 accuracy between a lag of $700 \mathrm{~ms}$ and $100 \mathrm{~ms}$ (cf. MacLean et al., 2012), was significantly larger in the alpha and beta range $(M=20 \%)$ than in the theta and gamma range $(M=7 \%), p<.001$. Neither the difference between alpha and beta, nor between theta and gamma, was significant at the $p<.05$ level. These conclusions were supported by the analyses given below.

Repeated-measures ANOVAs were conducted on the factors of Frequency and Lag for both first target accuracy (T1), and second target accuracy, contingent on successful first target detection (T2|T1). The $p$ values reported are GreenhouseGeisser corrected. T1 accuracy was strongly influenced by Frequency, but in a monotonic way: T1 accuracy decreased as the rate of stream presentation increased. The ANOVA indicated a significant main effect of frequency, $F(3,69)=$ 43.23, $p=.001, p \eta \mathrm{p}^{2}=.65$. There was also a main effect of lag, $F(2,46)=4.62, p=.017, p \eta p^{2}=.17$, reflecting $\mathrm{T} 1$

Table 1 Exact interval parameters for each entrainment frequency (ms)

\begin{tabular}{llll}
\hline Frequency & \multicolumn{2}{l}{ Lag } & \\
\cline { 2 - 4 } & $100 \mathrm{~ms}$ & $300 \mathrm{~ms}$ & $700 \mathrm{~ms}$ \\
\hline $36.0 \mathrm{~Hz}$ (Gamma) & 111 & 306 & 694 \\
$16.0 \mathrm{~Hz}$ (Beta) & 125 & 313 & 688 \\
$10.3 \mathrm{~Hz}$ (Alpha) & 97 & 292 & 681 \\
$6.26 \mathrm{~Hz}$ (Theta) & 160 & 319 & 639 \\
\hline
\end{tabular}

100 to 300 to $700 \mathrm{~ms}$ in each condition, with some additional temporal jitter to ensure that items were presented in phase in each condition (Colour figure online)

accuracy at Lag 700 being higher than at Lag 300, again at Lag 100. Most importantly, there was no hint of an interaction between Lag and Frequency, $F(6,138)=.34, p=.852, p \eta p^{2}=$ .01 .

Quite a different pattern emerged when we examined second target accuracy (T2|T1), as shown in Fig. 2. Here, the ANOVA indicated a significant interaction of Frequency $x$ Lag, $F(6,138)=4.30, p=.003, p \eta p^{2}=.16$, along with significant main effects of Frequency, $F(3,69)=33.54, p<$ $.001, p \eta p^{2}=.59$, and Lag, $F(2,46)=30.54, p<.001, p \eta p^{2}=$ .57. The interaction was examined in greater detail by computing the difference in accuracy between Lag 700 and Lag 100 for each frequency condition. This is a conventional measure of the magnitude of the $\mathrm{AB}$ when Lag 100 yields the lowest levels of accuracy (cf. MacLean \& Arnell, 2012). These differences were significantly greater than zero in each frequency condition $(p<.05)$, but Fisher's LSD comparisons between the conditions indicated that the $\mathrm{AB}$ was larger in the

Table 2 Number of stimuli between T1 and T2, for each Frequency $x$ Lag condition

\begin{tabular}{llll}
\hline Frequency & \multicolumn{2}{l}{ Lag } & \\
\cline { 2 - 4 } & $100 \mathrm{~ms}$ & $300 \mathrm{~ms}$ & $700 \mathrm{~ms}$ \\
\hline $36.0 \mathrm{~Hz}$ (Gamma) & 3 & 10 & 24 \\
$16.0 \mathrm{~Hz}$ (Beta) & 1 & 4 & 10 \\
$10.3 \mathrm{~Hz}$ (Alpha) & 0 & 2 & 6 \\
$6.26 \mathrm{~Hz}$ (Theta) & 0 & 1 & 3 \\
\hline
\end{tabular}


Table 3 Mean percentage correct for T1 and T2|T1 in Experiment 1

\begin{tabular}{llll}
\hline Frequency & \multicolumn{3}{l}{ Lag } \\
\cline { 2 - 4 } & $100 \mathrm{~ms}$ & $300 \mathrm{~ms}$ & $700 \mathrm{~ms}$ \\
\hline $\mathrm{T} 1$ & & & \\
$36.0 \mathrm{~Hz}$ (Gamma) & $67.5(3.69)$ & $69.3(3.60)$ & $70.6(3.07)$ \\
$16.0 \mathrm{~Hz}$ (Beta) & $85.2(2.51)$ & $87.0(2.18)$ & $86.9(2.71)$ \\
$10.3 \mathrm{~Hz}$ (Alpha) & $94.8(1.60)$ & $96.4(.95)$ & $96.6(.96)$ \\
$6.26 \mathrm{~Hz}$ (Theta) & $98.7(.46)$ & $98.7(.44)$ & $99.5(.27)$ \\
$\mathrm{T} 2 \mid \mathrm{T} 1$ & & & \\
$36.0 \mathrm{~Hz}$ (Gamma) & $65.4(4.22)$ & $68.9(3.71)$ & $74.4(3.89)$ \\
$16.0 \mathrm{~Hz}$ (Beta) & $58.8(4.29)$ & $64.8(3.74)$ & $82.5(3.51)$ \\
$10.3 \mathrm{~Hz}$ (Alpha) & $79.1(4.44)$ & $83.3(3.29)$ & $94.8(.98)$ \\
$6.26 \mathrm{~Hz}$ (Theta) & $94.6(1.32)$ & $99.0(.49)$ & $99.7(.22)$ \\
\hline
\end{tabular}

alpha and beta range than it was in the theta and gamma range, $F(1,69)=15.45, p<.001, p \eta p^{2}=.18$. More conservative Bonferroni-corrected comparisons between the four frequency conditions indicated the same pattern, with the $\mathrm{AB}$ in the theta condition being significantly smaller than in either the alpha or beta condition $(p<.01)$, and the beta condition having a significantly larger $\mathrm{AB}$ than the gamma condition $(p=$ .041). No other differences were significant.

One explanation to consider for the reduced attentional blink in the gamma frequency range is a floor effect; perhaps target identification is simply too impaired at these stream rates for the $\mathrm{AB}$ to be measured. We do not believe this is the case for two reasons. First, overall T1 and T2 accuracy in this condition were both well above the chance guessing level of 33\% ( $M=69 \%$ and $M=70 \%$, respectively). Second, the floor effect account would predict that the absence of the blink in the gamma condition, compared to the beta condition, is due to overall target identification difficulty. To test this, we subdivided the participants with a median split based solely on

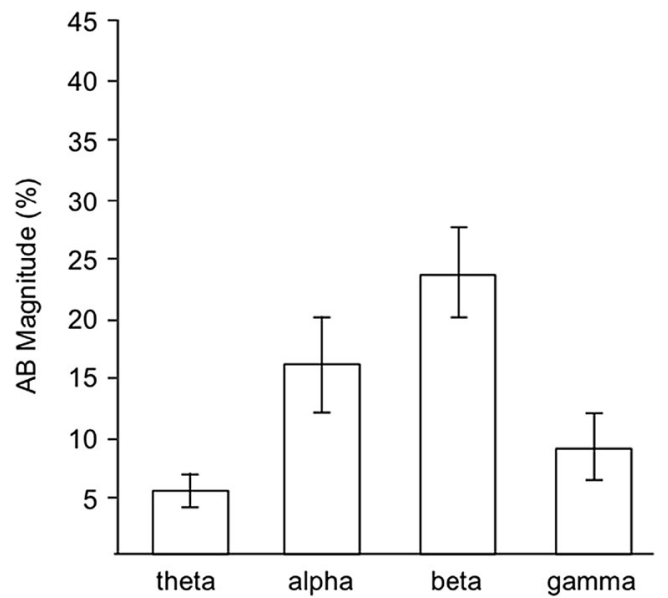

Fig. 2 Mean attentional blink (AB) in Experiment 1. AB is defined as T2 accuracy in Lag 700 minus T2 accuracy in Lag 100, conditional on correctly reporting $\mathrm{T} 1$ their T1 accuracy in the gamma condition. The top half of T1 gamma performers had a mean $\mathrm{T} 1$ accuracy of $82 \%$ in the gamma condition and $86 \%$ in the beta condition, which was not statistically different $(p=.29)$. Yet, these same participants did not show a significant $\mathrm{AB}$ in the gamma condition $(M=$ $3 \%, S D=8.7 \%, p=.27)$, but they did show a significant $\mathrm{AB}$ effect in the beta condition ( $M=15 \%, S D=23 \%, p=.04)$. Thus, the sharp reduction of $\mathrm{AB}$ in the gamma condition cannot be attributed to an overall increase in target identification difficulty.

There are also potential concerns about the reduced blink in the theta condition, given its high $\mathrm{T} 1$ accuracy and the overall slower rate of stimulus presentation, which may have differential masking effects on the T2 item (Brehaut, Enns, \& Di Lollo, 1999). To address these concerns we conducted a second experiment that addressed the potential concerns of floor effects on $\mathrm{T} 1$ accuracy, stream rate-concomitant effects on $\mathrm{T} 1$ accuracy, and differential masking effects on $\mathrm{T} 2$, at the same time as providing an opportunity to replicate the main finding in a substantially altered context.

\section{Experiment 2}

Experiment 2 was designed to address the potential concerns identified with our interpretation of Experiment 1, and to replicate the main finding of an increased blink in alpha and beta stream frequencies in another task context. Accordingly, we adjusted target visibility in advance via a luminance contrast manipulation in an effort to equate $\mathrm{T} 1$ performance across the four presentation rates. Secondly, we fixed the time interval between $\mathrm{T} 2$ and the subsequence distractor item for all rates of presentation, so that there would be equivalent levels of backward masking across all conditions.

\section{Method}

Experiment 2 was the same as Experiment 1 except as follows: Twelve participants (mean age: 21.8 years, nine female) participated in Experiment 2. Frequency order was counterbalanced across participants using a random Latin square design. The task was to report two letters presented among digits, in order to increase the depth of the attentional blink compared to Experiment 1. The stimuli were changed such that all distractors were randomly selected from the digits ' 1 ' through ' 9 '. Targets were selected from all 26 letters of the English alphabet, with the constraint that T1 and T2 would never have the same identity within a trial. Subjects were explicitly informed that ' $\mathrm{O}$ ' should be viewed as a letter, not the number zero. Participants were told they could report each target letter in any order, and responses matching either target were marked as correct at the position presented, regardless of the order in which the response was input. Participants were 
forced to make two different letter responses before the trial would continue. Chance levels of guessing for both T1 and T2 were $8 \%$.

All digits and letters except for T1 were light grey (RGB: [128 128 128]) presented on a black background. Courier font was used instead of Arial, due to poor spatial overlap between letters and numbers in Arial font (still $\sim 1^{\circ}$ visual angle). In order to better equate the overall accuracy of T2, the time between the onset of T1 and the following distractor was fixed at $97 \mathrm{~ms}$ for all conditions, and the distractor immediately preceding T2 (T2-1) in the Gamma condition was no longer presented (i.e. the T2-1 SOA changed from 28 to $56 \mathrm{~ms}$ ) to reduce the forward masking of $\mathrm{T} 2$. No other changes to the timing of the trial sequences were made (see Fig. 3).

In an effort to equate T1 accuracy across frequency and provide a more appropriate baseline to assess $\mathrm{T} 2$, the luminance of $\mathrm{T} 1$ relative to all other items (relative contrast) was manipulated for each frequency to achieve $80 \%$ T1 accuracy at Lag 700. Pilot data was used to estimate this threshold and set initial relative contrast values to $34 \%, 57 \%, 146 \%$, and $179 \%$ for the theta, alpha, beta and gamma conditions, respectively. Starting with these initial values, T1 contrast was adjusted in a 4-up, 1-down staircase procedure in increments of $20 \%$, based solely on Lag 700 performance, but applied uniformly to all lag conditions. The maximum T1 contrast was capped at $200 \%$ (RGB: [254 254 254]), due to the luminance limits of the display. The final threshold values across the twelve experimental participants matched well with the initial settings (M: $\left[\begin{array}{llllll}34 \% & 56 \% & 137 \% & 190 \%\end{array}\right]$ SD: [ $\left[\begin{array}{llll}13 \% & 17 \% & 32 \%\end{array}\right.$ $17 \%])$. This contrast manipulation was effective at maintaining equal T1 Lag 700 accuracy in all frequency conditions excepting the Gamma condition (M: [82\% 82\% 82\% 61\%], SD: [4\% 4\% 5\% 15\%]). In the gamma condition, nine of 12 participants had contrast thresholds at or above maximum allowed, resulting in reduced, though well above chance, T1 performance $($ chance $=8 \%)$.

Each participant completed 78 trials per block, for a total of 312 trials participant. All 26 were letters pseudo randomly selected to occur exactly once at the T1 and T2 positions for each Lag, within each frequency block.

\section{Results}

Table 4 shows the mean correct responses and standard errors for first target accuracy (T1) and for second target accuracy conditional on a correct response to T1 (T2|T1). Figure 4 shows the results for our experimental hypothesis, which replicates the main finding of Experiment 1: The magnitude of the attentional blink was larger in the alpha and beta conditions than in theta and gamma. These conclusions were supported by the following analyses.

A repeated-measures one-way ANOVA examined the influence of frequency on T1 accuracy and on the AB magnitude (calculated as the difference between T2|T1 performance at Lag 3 subtracted from T1 performance ${ }^{1}$ ). Lag 3 was used to index the $\mathrm{AB}$ in this experiment because this lag generally yielded the lowest levels of T2 accuracy in this experiment. This tends to occur when the task permits Lag-1 sparing to occur (for a review, see Visser, Bischof \& Di Lollo, 1999). Subsequent planned pairwise comparisons were then performed to determine the blink at each frequency relative to all other frequencies.

$\mathrm{T} 1$ accuracy was again significantly different between conditions, $F(3,33)=13.76, p=.001, \eta_{\mathrm{p}}{ }^{2}=.556$, this time driven by reduced accuracy in the gamma condition relative to the other conditions ( $p<.005$ for all comparisons between $36 \mathrm{~Hz}$ and all other frequencies). This means that some participants in the gamma conditions failed to reach $80 \%$ performance even with maximum contrast (200\%; see Table 4). Nevertheless, given that chance level performance in Experiment 2 was $8 \%$, the T1 contrast manipulation in Experiment 2 achieved the goal of dramatically reducing the number of randomly guessed $\mathrm{T} 1$ correct responses.

The attentional blink (T2|T1 accuracy at Lag 3 subtracted from T1) varied significantly with frequency. A one-way ANOVA indicated, $F(3,33)=4.62, p<.05, \eta_{\mathrm{p}}{ }^{2}=.296$, with subsequent one-tailed $t$ tests (see Table 5) revealing that all conditions exhibit an $\mathrm{AB}$ but $\mathrm{AB}$ magnitude is largest in the alpha and beta conditions. This outcome replicates the main finding of Experiment 1, but it does so while addressing the concerns in that experiment arising from larger concomitant differences in T1 accuracy and possible differential T2 masking effects across frequencies.

\section{Discussion}

The results of these two experiments are a compelling demonstration that the magnitude of the attentional blink - one of the most widespread demonstrations of information processing limitations in the human brain - is significantly larger when stimuli are presented at rates that trigger the prominent oscillatory frequencies in the human brain involved in neurally gating visual attention and conscious awareness: alpha and beta. It is equally important to note that finding a 'sweet spot' in the frequency at which the RSVP stream is presented is independent of general perceptual performance, that is, T1 perception. This was established in Experiment 2, where T1 accuracy was adjusted for each stream rate so as to ensure a

\footnotetext{
${ }^{1}$ Using $\mathrm{T} 1$ performance as the baseline ensures that differential recovery of the blink (T2 at Lag 7) does not inappropriately affect the calculation of blink magnitude. Although we attempted to equate T1 performance across frequencies, actual T1 performance (averaged over lags) for each frequency was used to calculate that particular frequency's AB. Note that we were unable to follow this procedure in Experiment 1 because of the differential effects of stream rate on T1 accuracy itself.
} 
Task is to report two letters amoung digits (Light Gray letters on a Dark Gray background)

$$
\begin{aligned}
& \text { 咅= Possible T1Locations I = Distractor Locations } \\
& \text { I=T2 Locations * * Report Targets Cue }
\end{aligned}
$$

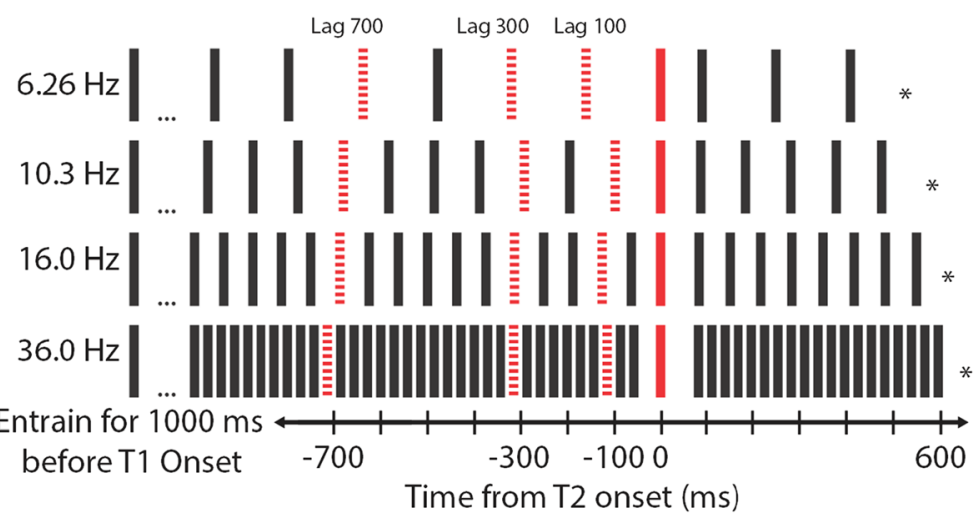

Fig. 3 The temporal sequence of events in Experiment 2. The task was to report two grey letters among grey digits presented against a black background. The luminance of T1 was adjusted for each participant in

high level, increasing confidence that the $\mathrm{AB}$ findings occurred under conditions where the first target was almost always reported successfully.

Although both experiments arrive at the same conclusion, Experiment 1 does suffer from potential methodological differences across frequency conditions that could give rise to alternative explanations of the data. Specifically, both $\mathrm{T} 1$ and T2 may have been differentially masked as a function of the frequencies of stimulus presentation. This was rectified in Experiment 2 where T2 masking was equated across frequencies. T1, although differentially masked as a consequence of the frequency of the stimulus stream, was adjusted for each individual to yield comparability across conditions. This design also addresses a potential problem in Experiment 1 stemming from the high baseline probability of guessing $\mathrm{T} 1$ when it was not seen $(33 \%)$. In Experiment 2, the probability of

\begin{tabular}{|c|c|c|c|}
\hline \multirow[t]{2}{*}{ Frequency } & \multicolumn{3}{|l|}{ Lag } \\
\hline & $100 \mathrm{~ms}$ & $300 \mathrm{~ms}$ & $700 \mathrm{~ms}$ \\
\hline \multicolumn{4}{|l|}{$\mathrm{T} 1$} \\
\hline $36.0 \mathrm{~Hz}$ (Gamma) & $57.1(13.7)$ & $63.8(13.6)$ & $60.9(14.5)$ \\
\hline 16.0 Hz (Beta) & $76.3(12.2)$ & $82.4(8.23)$ & $81.7(4.48)$ \\
\hline $10.3 \mathrm{~Hz}$ (Alpha) & $66.0(11.3)$ & $81.7(7.39)$ & $82.1(4.25)$ \\
\hline $6.26 \mathrm{~Hz}$ (Theta) & $73.7(17.0)$ & $84.3(9.48)$ & $82.4(3.99)$ \\
\hline \multicolumn{4}{|l|}{$\mathrm{T} 2 \mid \mathrm{T} 1$} \\
\hline $36.0 \mathrm{~Hz}$ (Gamma) & $69.59(13.22)$ & $44.66(20.16)$ & $73.73(14.24)$ \\
\hline $16.0 \mathrm{~Hz}$ (Beta) & $72.64(14.85)$ & $43.59(20.32)$ & $78.51(8.43)$ \\
\hline $10.3 \mathrm{~Hz}$ (Alpha) & $90.14(6.14)$ & $40.90(19.82)$ & 77.46 (11.79) \\
\hline $6.26 \mathrm{~Hz}$ (Theta) & $80.00(12.13)$ & $56.77(22.56)$ & $79.16(12.27)$ \\
\hline
\end{tabular}

Table 4 Mean percentage correct for T1 and T2|T1 in Experiment 2 an effort to equate T1 accuracy across frequency. Onset asynchrony between $\mathrm{T} 2$ and the $\mathrm{T} 2+1$ item was fixed at $100 \mathrm{~ms}$, to equate $\mathrm{T} 2$ backward masking across conditions (Colour figure online)

guessing an unseen item was much lower (8\%). Though the T1 accuracy in the gamma condition in Experiment 2 was somewhat reduced compared to the other conditions $(61 \%$ vs. $80 \%$ ), the probability that a correct $\mathrm{T} 1$ response was correct due to guessing was also much lower $(\sim 5 \%)$ than Experiment 1 (>20\%). Thus, in Experiment 2, differences in $\mathrm{T} 1 \mid \mathrm{T} 2$ accuracy between conditions cannot be accounted for by changes in $\mathrm{T} 2$ accuracy following unseen but "correct" $\mathrm{T} 1$ trials. Finally, we note that using T1 (rather than Lag 7) as the baseline in Experiment 2 does favour finding a smaller $\mathrm{AB}$ in the gamma condition. We chose this baseline as Lag 7 performance is inherently subject to fluctuations in the recovery of the $\mathrm{AB}$ as observed in Experiment 1; unlike T1, which is an $\mathrm{AB}$-independent measure of stimulus recognition. Using Lag 7 to calculate $\mathrm{AB}$ magnitude in Experiment 2 does not show

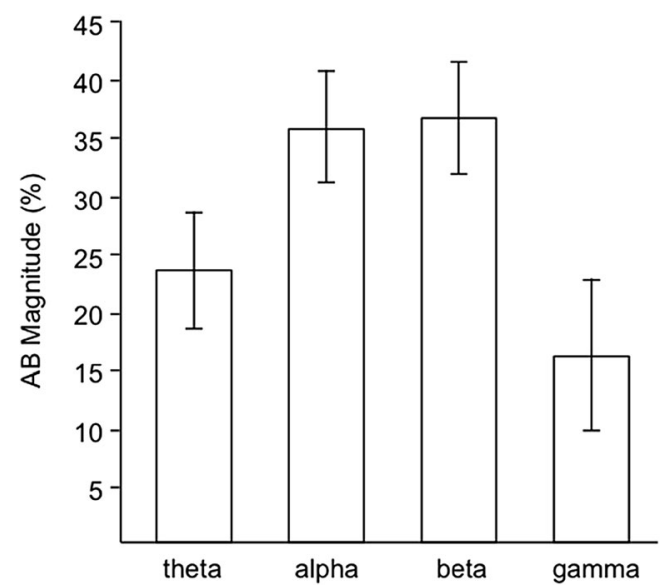

Fig. 4 Mean attentional blink $(\mathrm{AB})$ in Experiment 2. $\mathrm{AB}$ is defined as the difference between T1 accuracy and T2 accuracy at Lag 300, conditional on correctly reporting $\mathrm{T} 1$ 
Table 5 Planned pairwise $t$ tests (one-tailed) comparing AB magnitude across frequencies in Experiment 2

\begin{tabular}{llll}
\hline & Theta & Alpha & Beta \\
\hline Alpha & $p<.05$ & & \\
Beta & $p<.05$ & $n s$ & \\
Gamma & $n s$ & $p<.05$ & $p<.05$ \\
\hline
\end{tabular}

the expected difference in gamma from the alpha-beta conditions but leaves all other results unaffected.

The finding that the attentional blink is frequency specific points to a long neglected factor in theories of the $\mathrm{AB}$, which have all claimed the inter-target lag to be the most important predictor of second target accuracy. Theories of the AB must now account for these frequency-specific effects. We believe such effects point to underlying neural circuitry that opens and closes access to conscious perception, potentially both endogenously and exogenously. Previous reports of exogenous and endogenous alpha playing a role in the $\mathrm{AB}$ have been made by Arnell and colleagues, though the relationship of their oscillatory measure (event-related desynchronization) to the present measure remains to be determined (MacLean \& Arnell, 2011; MacLean et al., 2012).

We hasten to point out that the present findings need to be examined under the full spotlight of the many $A B$ approaches and findings that populate the extant literature, including those scarce reports that find an $\mathrm{AB}$ for stimulus streams presented outside the alpha-beta 'sweet spot' found in the present report. As noted in the introduction, one class of theories for the $\mathrm{AB}$ cites the interval between targets as the key determinant, since this interval limits the time available for the first target to be consolidated into working memory (Chun \& Potter, 1995). A competing set of theories also sees this interval as the key determinant, albeit for different a reason. For them the interval represents the limited time available to regain control over an attentional filter that has inadvertently been waylaid by distractor items in the stream (Di Lollo et al., 2005; Olivers \& Meeter, 2008). The present finding should help sharpen each of these theoretical positions, since it implies that the limiting factors of working memory consolidation or control over an attentional filter are influenced by specific brain oscillations, not only by the mere passage of time.

We also contend out that our conclusion is not undermined by the existence of the so-called skeletal AB (cf. Duncan, Ward, \& Shapiro, 1994) where an AB is found when only the two targets are presented, each masked, separated by the appropriate temporal interval. Although no pre-T1 stimuli are presented to entrain a given frequency as in the present experiment, it is well known that the brain produces endogenous frequencies that facilitate performance on cognitive tasks. By our account (cf. Hanslmayr et al., 2011) the identification of T1 causes an increase in alpha oscillatory activity that subsequently closes the thalamocortical input channel to result in an AB. A similar account based solely on behavioural data was suggested by Chun and Potter (1995). Thus we propose that a pre-T1 stimulus stream in a frequency other than alpha or beta prevents this synchronization, in turn preventing the closure of the perceptual channel and attenuating the $\mathrm{AB}$. Further experiments measuring oscillations will be required to verify this account.

An interesting finding is that in Experiment 2 much larger Lag-1 sparing was observed in the alpha band than any other band (all bands showed some, but in alpha T2/ $\mathrm{T} 1$ accuracy was actually higher by $13 \%$ at Lag 1 than in Lag 7). Although this may be a clue to understanding the role of alpha oscillations in the $\mathrm{AB}$, we are hesitant to make too much of this observation as (1) differences in Lag-1 sparing have been attributable to many different causes (e.g. Visser et al., 1999), and (2) calculations based on Lag 7 are subject to differential rates of $A B$ recovery and hence must be made with caution.

Although these findings may be provocative from a cognitive-behavioural perspective, they are in line with recent electrophysiological studies on alpha/beta oscillations. A now well accepted framework states that increased alpha/beta oscillations reflect inhibition of neural assemblies (Klimesch, Sauseng, \& Hanslmayr, 2007; Jensen \& Mazaheri, 2010). Accordingly, visual stimuli are more often missed when presented during periods of high alpha/beta oscillatory as compared to periods of low alpha/beta activity (van Dijk, Schoffelen, Oostenveld, \& Jensen, 2008). These, and other, findings lead to the conclusion that increased alpha/beta activity represents a worst-case condition for visual perception, which could explain why the $\mathrm{AB}$ is larger when the brain is driven or naturally oscillating at these frequencies (Shapiro \& Hanslmayr, 2014). Together, our results suggest that access to conscious awareness is mediated by one of the brain's dominant frequencies (alpha), as has been shown in hundreds of prior studies, and by only one other frequency (beta); a frequency often revealing outcomes paralleling those of alpha (Hanslmayr et al., 2011). Although the hypothesis we favour awaits confirmation by EEG analysis, the data are consistent with this hypothesis and supported by the growing body of literature suggesting that brain oscillations play an important role in cognitive information processing.

Author note This research was supported by the following research granting agencies: KL Shapiro (Human Frontiers of Science Programme); JT Enns (NSERC Discovery Grant, Canada and Adjunct 
Professor, Department of Psychology, University of Western Australia); Simon Hanslmayr (German Research Foundation, DFG HA5622/1-1).

Open Access This article is distributed under the terms of the Creative Commons Attribution 4.0 International License (http:// creativecommons.org/licenses/by/4.0/), which permits unrestricted use, distribution, and reproduction in any medium, provided you give appropriate credit to the original author(s) and the source, provide a link to the Creative Commons license, and indicate if changes were made.

\section{References}

Arnell, K. M., Howe, A. E., Joanisse, M. F., \& Klein, R. M. (2006), Relationships between attentional blink magnitude, RSVP target accuracy, and performance on other cognitive tasks. Memory \& Cognition, 34(7), 1472-1483.

Berger, H. (1929). Über das elektrenkephalogramm des menschen [On the human encephalogram]. Archiv für Psychiatrie und Nervenkrankheiten, 87, 527-570.

Brehaut, J. C., Enns, J. T., \& Di Lollo, V. (1999). Visual masking plays two roles in the attentional blink. Perception \& Psychophysics, 61(7), 1436-1448.

Buzsáki, G., \& Draguhn, A. (2004). Neuronal oscillations in cortical networks. Science, 304, 1926-1929.

Chun, M. M., \& Potter, M. C. (1995). A two-stage model for multiple target detection in rapid serial visual presentation. Journal of Experimental Psychology: Human Perception and Performance, 21(1), 109-127.

Di Lollo, V., Kawahara, J.-I., Ghorashi, S. M., \& Enns, J. T. (2005). The attentional blink: Resource depletion or temporary loss of control? Psychological Research, 69, 191-200.

Duncan, J., Ward, R., \& Shapiro, K. (1994). Direct measurement of attentional dwell time in human vision. Nature, 369, 313-315.

Hanslmayr, S., Gross, J., Klimesch, W., \& Shapiro, K. L. (2011). The role of alpha oscillations in temporal attention. Brain Research Reviews, 67, 331-343.

Hanslmayr, S., Staudigl, T., \& Klimesch, W. (2007). Prestimulus oscillations predict visual perception performance between and within subjects. NeuroImage, 37(4), 1465-1473. doi:10.1016/j.neuroimage. 2007.07.011

Herrmann, C. S. (2001). Human EEG responses to 1-100 Hz flicker: Resonance phenomena in visual cortex and their potential correlation to cognitive phenomena. Experimental Brain Research, 137, 346-353. doi: $10.1007 / \mathrm{s} 002210100682$

Jensen, O., \& Mazaheri, A. (2010). Shaping functional architecture by oscillatory alpha activity: Gating by inhibition. Frontiers in Human Neuroscience, 4. doi:10.3389/fnhum.2010.00186
Klimesch, W., Sauseng, P., \& Hanslmayr, S. (2007). EEG alpha oscillations: The inhibition-timing hypothesis. Brain Research Reviews, 53(1), 63-88. doi:10.1016/j.brainresrev.2006.06.003

Luck, S. J., Vogel, E. K., \& Shapiro, K. L. (1996). Word meanings can be accessed but not reported during the attentional blink. Nature, 382, 616-618.

MacLean, M. H., \& Arnell, K. M. (2011). Greater attentional blink magnitude is associated with higher levels of anticipatory attention as measured by alpha event-related desynchronization (ERD). Brain Research, 1387, 99-107. doi:10.1016/j.brainres.2011.02.069

MacLean, M. H., \& Arnell, K. M. (2012). A conceptual and methodological framework for measuring and modulating the attentional blink. Attention, Perception \& Psychophysics, 74(6), 1080-1097. doi:10.3758/s13414-012-0338-4

MacLean, M. H., Arnell, K. M., \& Cote, K. A. (2012). Resting EEG in alpha and beta bands predicts individual differences in attentional blink magnitude. Brain and Cognition, 1-14. doi:10.1016/j.bandc. 2011.12.010

Mathewson, K. E., Prudhomme, C., Fabiani, M., Beck, D. M., Lleras, A., \& Gratton, G. (2012). Making waves in the stream of consciousness: Entraining oscillations in EEG alpha and fluctuations in visual awareness with rhythmic visual stimulation. Journal of Cognitive Neuroscience, 24(12), 2321-2333.

Mima, T., Oluwatimilehin, T., Hiraoka, T., \& Hallett, M. (2001). Transient interhemispheric neuronal synchrony correlates with object recognition. Journal of Neuroscience, 21, 3942-3948.

Olivers, C. N. L., \& Meeter, M. (2008). A boost and bounce theory of temporal attention. Psychological Review, 115, 836-863.

Pletzer, B., Kerschbaum, H., \& Klimesch, W. (2010). When frequencies never synchronize: The golden mean and the resting EEG. Brain Research, 1335, 91-102.

Raymond, J., Shapiro, K., \& Arnell, K. (1992). Temporary suppression of visual processing in an RSVP task: An attentional blink? Journal of Experimental Psychology: Human Perception and Performance, $18,849-860$

Scheeringa, R., Fries P., Petersson K. M., Oostenveld, R., Grothe, I., Norris, D. G., ... Bastiaansen, M. C. (2011). Neuronal dynamics underlying high- and low-frequency EEG oscillations contribute independently to the human BOLD signal. Neuron, 69(3), 572-83. doi:10.1016/j.neuron.2010.11.044

Shapiro, K. L., \& Hanslmayr, S. (2014). The role of brain oscillations in temporal attention. In A. C. Nobre \& S. Kastner (Eds.), Oxford handbook of attention. Oxford: Oxford University Press. doi:10. 1093/oxfordhb/9780199675111.013.037

van Dijk, H., Schoffelen, J.-M., Oostenveld, R., \& Jensen, O. (2008). Prestimulus oscillatory activity in the alpha band predicts visual discrimination ability. Journal of Neuroscience, 28, 1816-1823.

Visser, T. A., Bischof, W. F., \& Di Lollo, V. (1999). Attentional switching in spatial and nonspatial domains: Evidence from the attentional blink. Psychological Bulletin, 125(4), 458. 